\section{Contrast-enhanced ultrasonography of the liver using SonoVue}

\author{
Giovanna Ferraioli ${ }^{1}$, Maria Franca Meloni ${ }^{2}$ \\ 'Ultrasound Unit, Infectious Diseases Department, Fondazione IRCCS Policlinico San Matteo, \\ University of Pavia, Pavia; ${ }^{2}$ Interventional Ultrasound, Casa di Cura Igea, Milano, Italy
}

This review focuses on the use of contrast-enhanced ultrasonography (CEUS) with SonoVue (Bracco Imaging) for the diagnosis of focal liver lesions (FLLS), guidance during ablative treatment, and follow-up of liver tumors. Multicenter trials have shown that the performance of CEUS for the characterization of FLLS is similar to that of contrast-enhanced computed tomography or contrast-enhanced magnetic resonance imaging. CEUS with SonoVue has been effectively used for guiding the percutaneous treatment of malignant liver tumors that are invisible or poorly visualized in traditional gray-scale ultrasonography. Postprocedural CEUS may be used to detect and retreat residual viable tissue in the same ablative session. The use of CEUS for the assessment of diffuse liver disease is also discussed.

Keywords: Liver; Contrast-enhanced ultrasound; Liver tumors; Carcinoma, hepatocellular; Liver metastasis

\section{Introduction}

With the widespread use of ultrasonography (US) as an imaging technique, due to its noninvasiveness, repeatability, and relatively low cost, the rate of incidentally detected focal liver lesions (FLLS) has increased. In asymptomatic and healthy persons without risk factors, these lesions are usually benign; therefore, it is of the utmost importance to arrive at a definitive diagnosis in a way that avoids more expensive studies that are not free of risks $[1,2]$. Nonetheless, early detection and characterization of primary and metastatic liver cancers could allow more effective treatments, thereby improving patients' survival. Contrast-enhanced ultrasound (CEUS) is a real-time dynamic imaging technique that plays an important role in this setting because it permits the characterization of FLLs due to its ability to demonstrate the vascularity of the tumor.

Few US contrast agents are available on the market for performing CEUS studies. In Europe, the revision is accurate used is SonoVue (Bracco Imaging, Milan, Italy). Unlike computed tomography (CT) or magnetic resonance (MR) contrast media, SonoVue is a purely intravascular agent, without an interstitial extravascular phase, allowing the dynamic detection of microcirculation.

SonoVue is made up of microbubbles $(2-10 \mu \mathrm{m})$ with a shell of phospholipids that are filled with sulfur hexafluoride gas. The contrast agent, which is confined in the vessels after the injection, presents a high reflectivity with a low mechanical index and allows the visualization of the tiny vessels in the capillary bed, thus permitting the dynamic detection of capillary microvascularization.

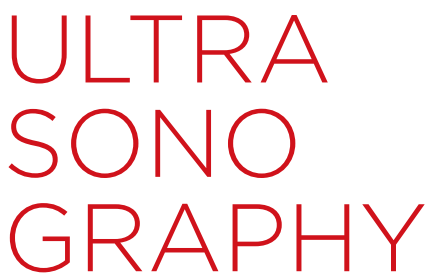

REVIEW ARTICLE

https://doi.org/10.14366/usg. 17037 pISSN: 2288-5919 • elSSN: 2288-5943 Ultrasonography 2018;37:25-35

Received: May 10, 2017

Revised: June 19, 2017

Accepted: July 12, 2017

Correspondence to: Giovanna Ferraioli, MD, Ultrasound Unit, Infectious Diseases Department, Fondazione IRCCS Policlinico San Matteo, University of Pavia, Via Taramelli 5, Pavia 27100, Italy Tel. +39-0382-502799 Fax. +39-0382-502296 E-mail: giovanna.ferraioli@unipv.it

This is an Open Access article distributed under the terms of the Creative Commons Attribution NonCommercial License (http://creativecommons.org/ licenses/by-nc/3.0/) which permits unrestricted noncommercial use, distribution, and reproduction in any medium, provided the original work is properly cited.

Copyright @ 2018 Korean Society of Ultrasound in Medicine (KSUM)

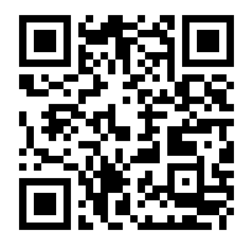

How to cite this article:

Ferraioli G, Meloni MF. Contrast-enhanced ultrasonography of the liver using SonoVue. Ultrasonography. 2018 Jan;37(1):25-35. 
The contrast agent is not excreted by the kidneys, and it can therefore be used in patients with impaired renal function in whom contrast-enhanced CT (CE-CT) or contrast-enhanced MR (CE-MR) is contraindicated. The safety of SonoVue has been retrospectively assessed in a series of 23,188 investigations performed in adult subjects: no fatal events occurred and the overall rate of serious adverse events was $0.0086 \%$ [3].

The liver has a dual blood supply, from the portal vein and the hepatic artery, which gives rise to three US contrast vascular phases: the arterial phase that starts within 20 seconds after the injection and continues for 30-45 seconds, followed by the portal venous phase that ends at 120 seconds, and then the late phase that lasts up to the clearance of the US contrast agent from the circulation (usually 6 minutes) [4].

Special perfusion software also allows blood volume-based parameters to be measured (e.g., maximum intensity and area under the curve) and flow rate-based parameters (e.g., mean transit time and time to peak) [5].

This review focuses on the use of CEUS with SonoVue for the diagnosis of FLLs, guidance during ablative treatments, and post-ablation follow-up of liver tumors. The use of CEUS for the assessment of diffuse liver disease is discussed as well.

\section{Diagnosis of Focal Liver Lesions}

SonoVue is a form of ultrasound contrast that has been widely used in Europe and parts of Asia for the characterization of FLLS for almost 15 years, and its performance has been evaluated in numerous studies.

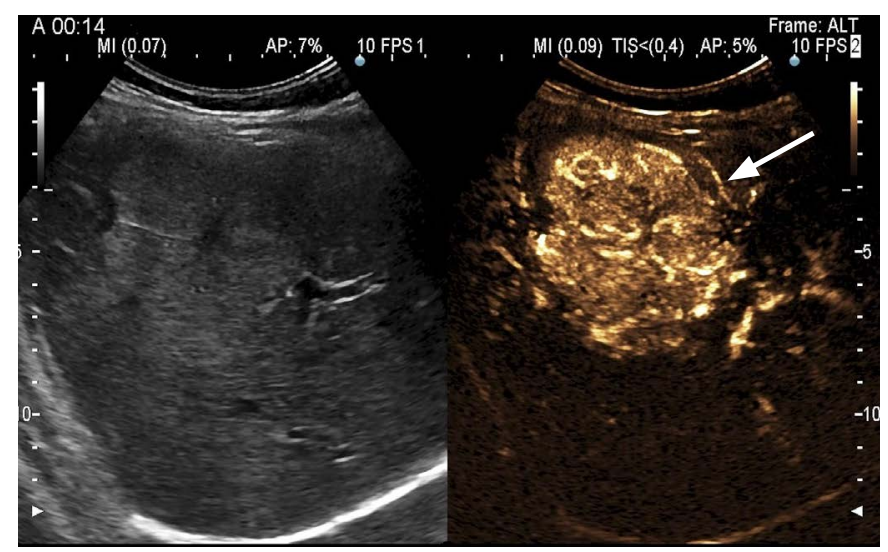

A
The European Federation of Societies for Ultrasound in Medicine and Biology (EFSUMB) and the World Federation for Ultrasound in Medicine and Biology (WFUMB), together with representatives of other scientific bodies, have produced guidelines for the use of CEUS in the liver that report the enhancement patterns of benign and malignant FLLS after intravenous administration of the US contrast agent [4]. Sustained enhancement in the portal venous and late phases, without washout signs, is typically observed in almost all solid benign liver lesions, whereas hypo-enhancement in the late phases, corresponding to washout, is the key finding of malignancies (Figs. 1, 2) [4].

Multicenter trials have shown that the performance of CEUS for the characterization of FLLs is similar to that of CE-CT or CE-MR [69]. A meta-analysis including 8,147 FLLs showed that the overall sensitivity and specificity of CEUS for the diagnosis of malignant liver lesions were $93 \%$ (95\% confidence interval [Cl], 91 to 95 ) and $90 \%$ (95\% Cl, 88 to 92$)$, respectively [10]. The meta-analysis comprised 45 studies, 35 (77.8\%) performed with SonoVue and the other 10 with other contrast agents: Levovist in eight studies, Optison in one study, and Sonazoid in one study. Significant heterogeneity was found across studies; however, the sources of heterogeneity could not be sufficiently identified. Nonetheless, a sub-analysis revealed that there was no significant difference when evaluating studies using histology for all FLLs, when comparing high-quality and lowquality studies, or blinded versus nonblinded studies.

Westwood et al. [11] conducted a study, commissioned by the Secretary of Health of the United Kingdom, comparing the clinical effectiveness and cost-effectiveness of CEUS using SonoVue with that of CE-CT and CE-MR for the assessment of adults with FLLs in

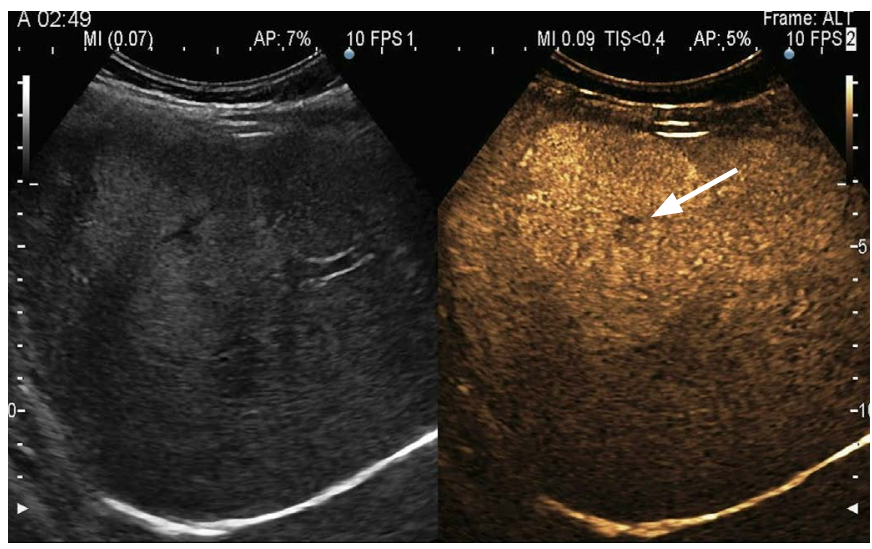

B

Fig. 1. Focal nodular hyperplasia in a 36-year-old woman without a history of liver disease.

A. A $4.8 \times 5.1-\mathrm{cm}$ incidental focal liver lesion in segment 5 shows a spoke-wheel appearance (arrow) after SonoVue injection. In the early arterial phase, there was centrifugal enhancement. B. In the late phase, the lesion exhibits sustained enhancement except a central scar (arrow). 
whom previous liver imaging studies were inconclusive. The authors reported that CEUS could provide similar diagnostic performance to other imaging modalities for the assessment of FLLs. Economic analyses indicated that CEUS was a cost-effective replacement for CE-MR. The use of CEUS instead of CE-CT was considered costeffective in the characterization of nodules detected during the

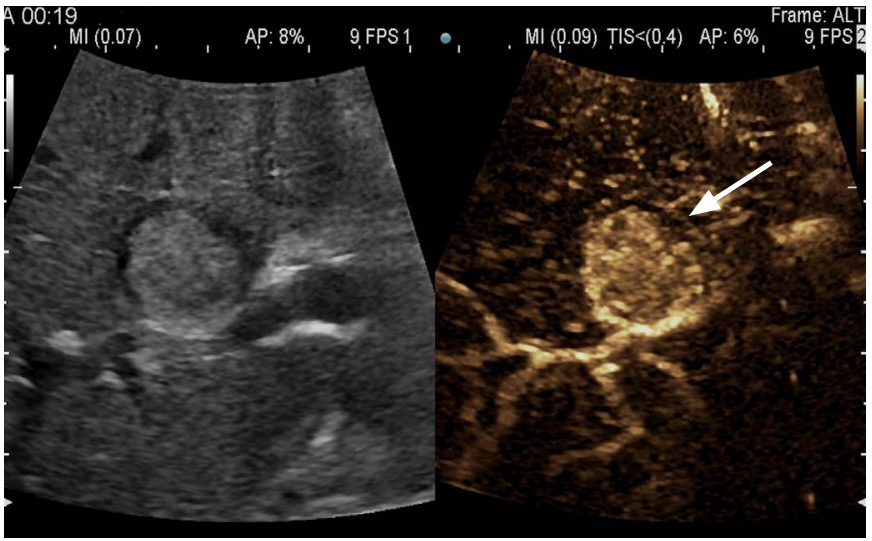

A

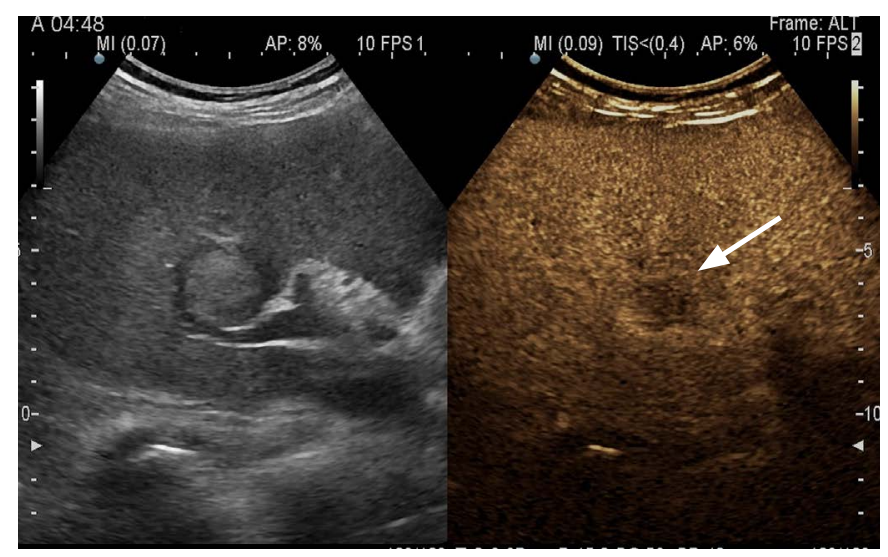

C

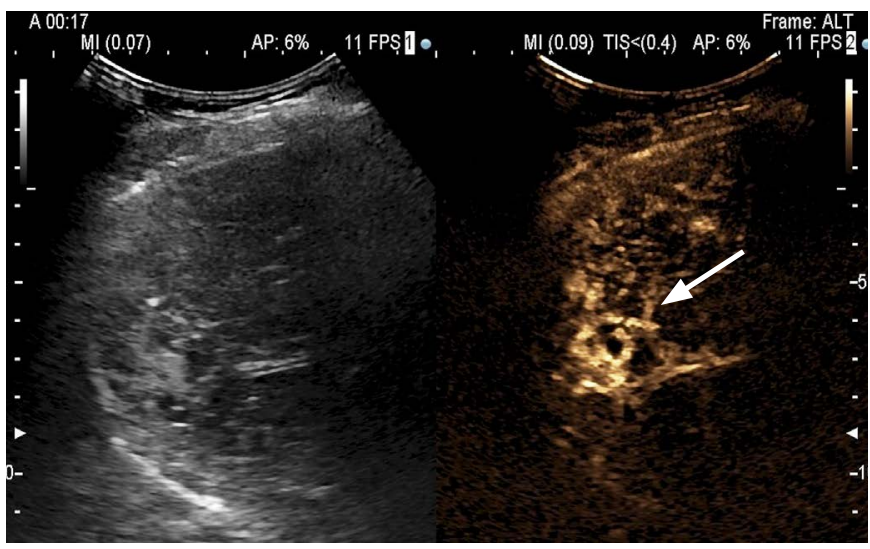

A

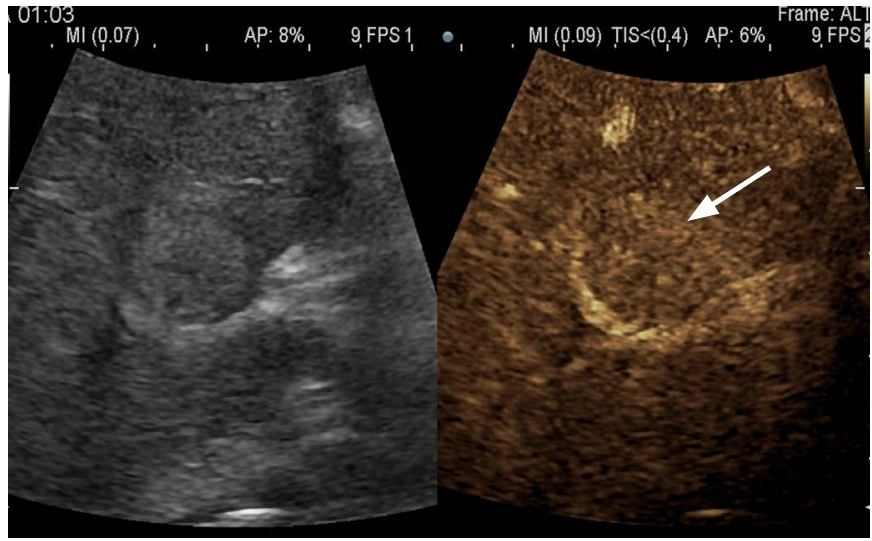

B

Fig. 2. Hepatocellular carcinoma in a 69-year-old cirrhotic man with alcohol addiction and chronic hepatitis $B$.

A. During surveillance, a $2.5-\mathrm{cm}$ nodule was detected in segment 5. In the early arterial phase, the lesion becomes hyper-enhancing (arrow) with respect to the surrounding liver. B. The nodule is isoenhancing (arrow) in the portal venous phase. C. The nodule showed a slow and mild washout and becomes hypoechoic (arrow) in the late phase.

Fig. 3. A 68-year-old woman with liver cirrhosis and a hyperechoic nodule in segment 7.

A. In contrast-enhanced ultrasonography, the nodule shows a globular enhancement (arrow) in the arterial phase. This feature was typical of hemangioma. B. In the late phase, the entire nodule is hyper-enhancing (arrow) with respect to the adjacent liver. It was categorized as LR-1 (a definitely benign lesion). 
surveillance of cirrhotic patients and for the characterization of incidentally detected FLLs, with similar costs and effects for the detection of liver metastases from colorectal cancer.

A study that involved three centers and 485 subjects with 575 incidentally detected FLLs reported that CEUS correctly differentiated (benign vs. malignant) 559 of 575 lesions (97.2\%), with $98.1 \%$ sensitivity and $95.7 \%$ specificity, and saved 175.39 Euros per patient [12]. In this study, CE-CT or CE-MR was the reference standard.

In patients without known pathologies, a meta-analysis that included 21 studies showed that the diagnostic accuracy of CEUS with SonoVue was not significantly different from that of CE-CT or CE-MR [13]. The sensitivity and specificity, respectively, were $88 \%$ (95\% Cl, 87 to 90$)$ and $81 \%(95 \% \mathrm{Cl}, 79$ to 84$)$ for CEUS, $90 \%$ (95\% $\mathrm{Cl}, 88$ to 92$)$ and $77 \%(95 \% \mathrm{Cl}, 71$ to 82$)$ for $\mathrm{CE}-\mathrm{CT}$, and $86 \%(95 \%$ $\mathrm{Cl}, 83$ to 88$)$ and $81 \%(95 \% \mathrm{Cl}, 76$ to 85$)$ for CE-MR.

In a multicenter study that enrolled 1,349 patients at 14 US

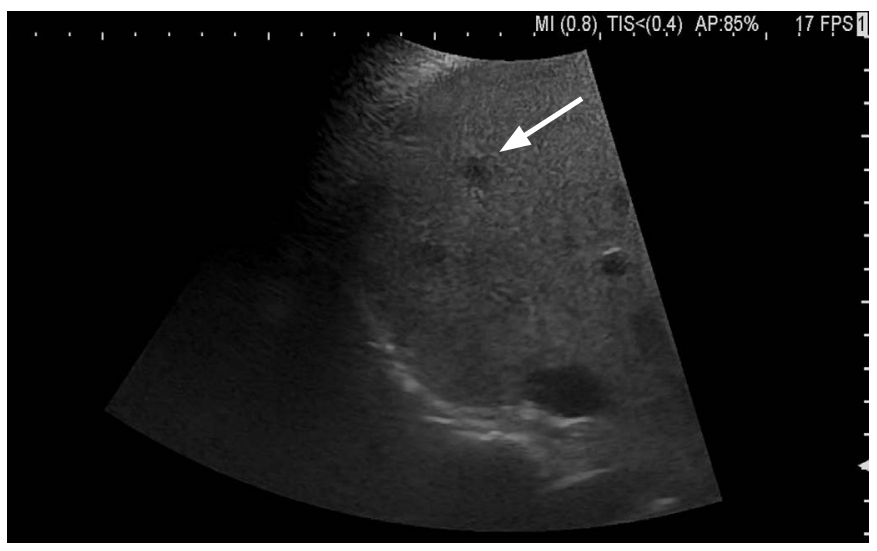

A

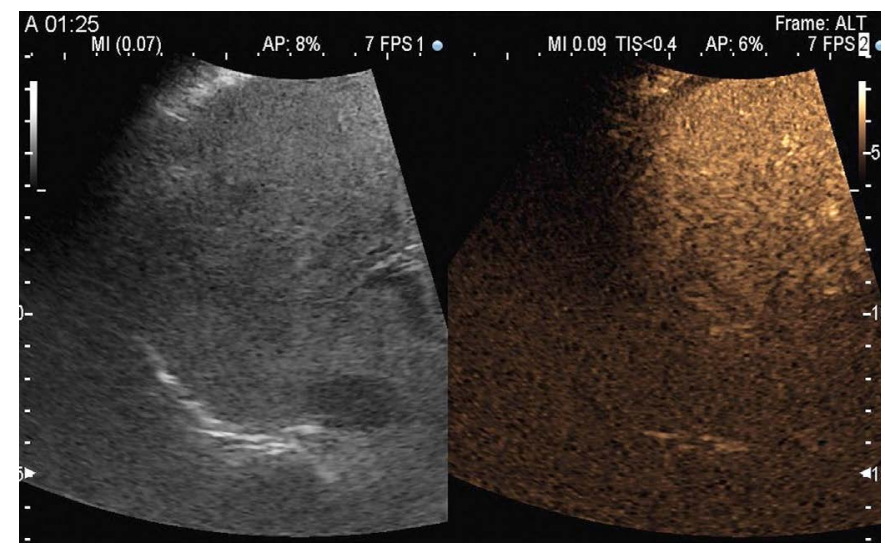

C

Fig. 4. A 64-year-old man with chronic hepatitis C.

A. A 9-mm hypoechoic nodule (arrow) is seen in segment 5 liver. B. In the arterial phase, the nodule shows enhancement similar to the surrounding liver parenchyma. C. In the portal venous phase, there is no washout of the contrast material distinguished from background parenchyma. D. In the late phase, the nodule looks still iso-enhancing. It was classified as LR-2 (probably benign).
B

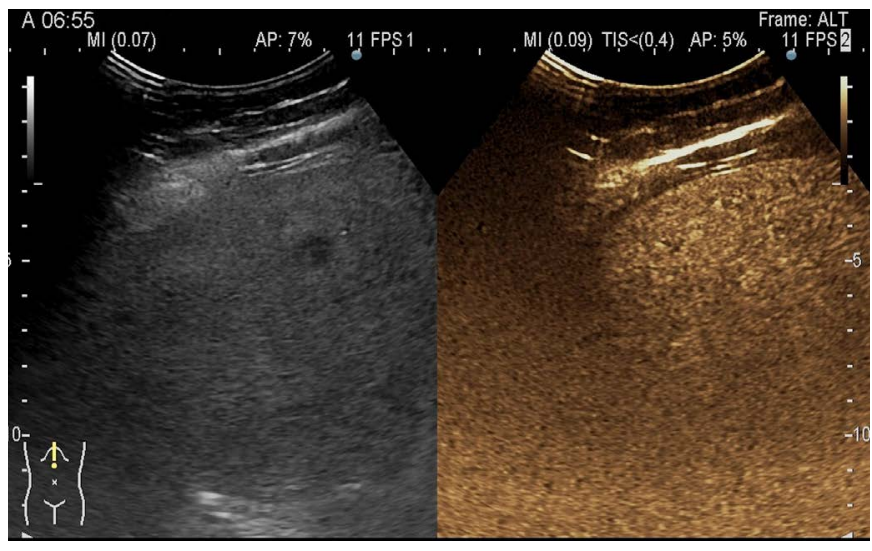

D centers, the overall diagnostic accuracy of CEUS in differentiating benign from malignant FLLs, compared to histology in more than $70 \%$ of cases or to CE-CT or CE-MR in the remaining cases, was $90.3 \%$ [7]. For the diagnosis of a malignant tumor, CEUS had a $92.3 \%$ positive predictive value and a $95.1 \%$ negative predictive value.

Unusual patterns of focal fat deposition may mimic malignancies and cause diagnostic challenges on imaging [14]. It has been reported that in the detection of liver metastases in cancer patients with geographic liver fatty infiltration on US, CEUS with SonoVue had $100 \%$ sensitivity and $97.1 \%$ specificity [15]. No statistically significant difference was found between CEUS and CE-MR in the detection of FLLS.

CEUS is a real-time dynamic imaging technique that can be used when CE-MR or CE-CT is inconclusive or contraindicated. In fact, this continuous real-time imaging modality may allow detecting the transient enhancement of a lesion that could be missed by other

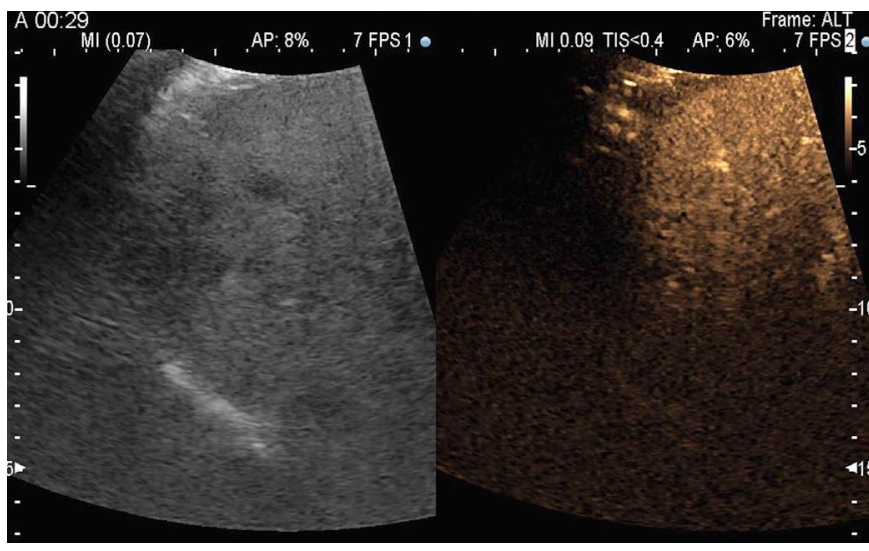


imaging modalities [16].

In the guidelines for management of hepatocellular carcinoma ( $\mathrm{HCC}$ ) produced by the American Association for the Study of Liver Disease and by the European Association for the Study of the Liver, CEUS was removed from the diagnostic algorithm for nodules in cirrhosis $[17,18]$. This choice was based on a study that enrolled a relatively low number of patients recruited over a long time and showed false-positive HCC diagnoses in patients with intrahepatic cholangiocellular carcinoma [19].

However, the use of CEUS for the characterization of nodules in cirrhotic livers is recommended by the Italian Association for the Study of the Liver and the Italian National Institute of Health's Sistema Nazionale Linee Guida $[20,21]$. The British National Institute for Health and Clinical Excellence recommends the use of SonoVue for characterizing FLLs in adults whose cirrhosis is being monitored if CE-MR is not clinically appropriate, is not accessible or is not acceptable to the person, and when an unenhanced US scan is inconclusive [22].

In a retrospective study performed on 282 cirrhotic patients with 34 benign and 248 histologically proven malignant FLLs, a washout time $>55$ seconds identified patients with HCC with the highest level of accuracy (92.7\%), whereas a washout time $\leq 55$ seconds correctly identified the vast majority of the non-HCC malignancies (100\% sensitivity, $98.2 \%$ specificity, $98.3 \%$ diagnostic accuracy) [23].

In a recent multicenter prospective trial, 442 cirrhotic patients were enrolled to assess the diagnostic performance of CE-CT, CE$M R$, and CEUS, alone or in combination, for the diagnosis of HCC 10 to $30 \mathrm{~mm}$ in size [24]. CEUS, CE-CT, and CE-MR were performed in all patients within a month. This trial showed that the sensitivity of CEUS was low but the specificity was $92.9 \%$, significantly higher than CE-CT (76.8\%) and CE-MR (83.2\%) for nodules 10-20 mm

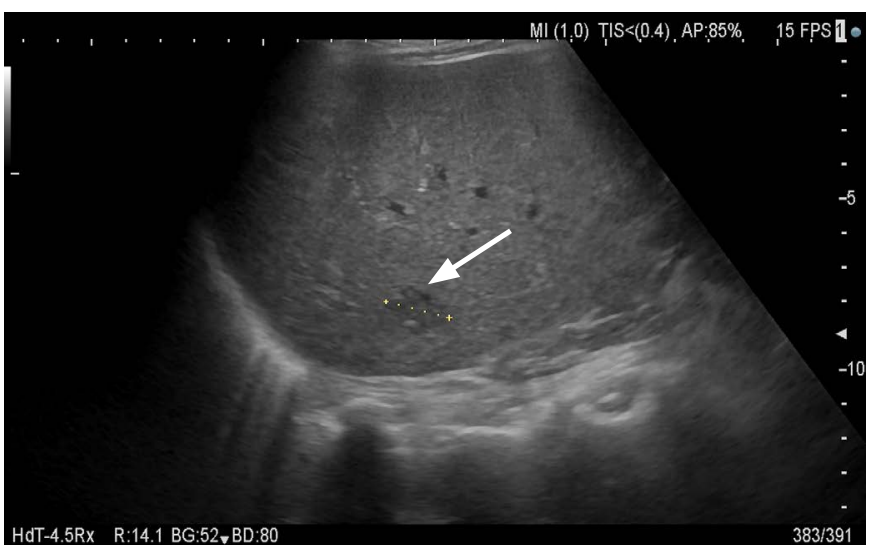

A

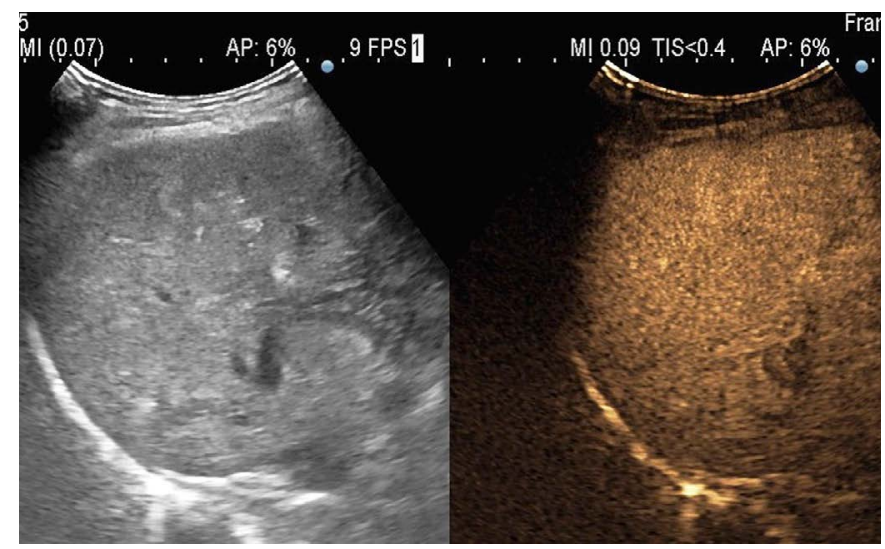

C

Fig. 5. A 62-year-old man with alcoholic liver cirrhosis.

A. During surveillance, a 19-mm nodular lesion (arrow) is seen in segment 7. B. The nodule is iso-enhancing with respect to the surrounding liver parenchyma in the arterial phase. C. In the late phase, 3 minutes 45 seconds after the administration of the ultrasound contrast, the nodule looks still iso-enhancing. D. Later, at 4 minutes 28 seconds, mild washout (arrows) is detected. The nodule was classified as LR-3 (an intermediate probability of hepatocellular carcinoma). Ultrasonography-guided biopsy of the nodule confirmed a high-grade dysplastic nodule. 
in size. The best sequential approach combined CE-MR and CEUS; thus, the authors suggested that CEUS could play a role in a 2-step algorithm for the noninvasive diagnosis of HCC.

The Liver Imaging Reporting and Data System using CEUS (CEUS LI-RADS) for the diagnosis of HCC in patients at risk was recently developed to improve the consensus and standardization regarding diagnostic performance, interpretation, and reporting [25]. CEUS LIRADS is an algorithm that classifies nodules from LR-1, a definitely benign lesion, through LR-5, which is definitely HCC (Figs. 3-8). A conclusive diagnosis of HCC is made when the nodule is $\geq 10 \mathrm{~mm}$ in size and the whole nodule or part of it shows hyperenhancement in the arterial phase followed by late ( $\geq 60$ seconds) and mild washout. These criteria virtually eliminate the risk of misdiagnosing cholangiocarcinoma [25].

Due to the lack of a panoramic view, CEUS is not recommended for staging liver malignancies; however, the technique is highly accurate in the characterization of FLLs. In CEUS studies, liver metastases show a very rapid and marked washout, meaning that the conspicuity and detection increase in the late phase (Fig. 9), allowing the evaluation of the whole liver. In the setting of

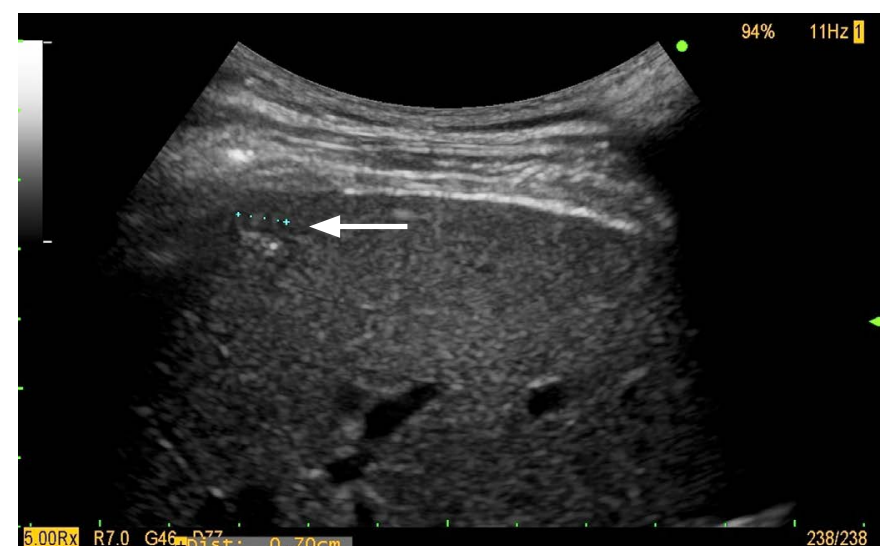

A

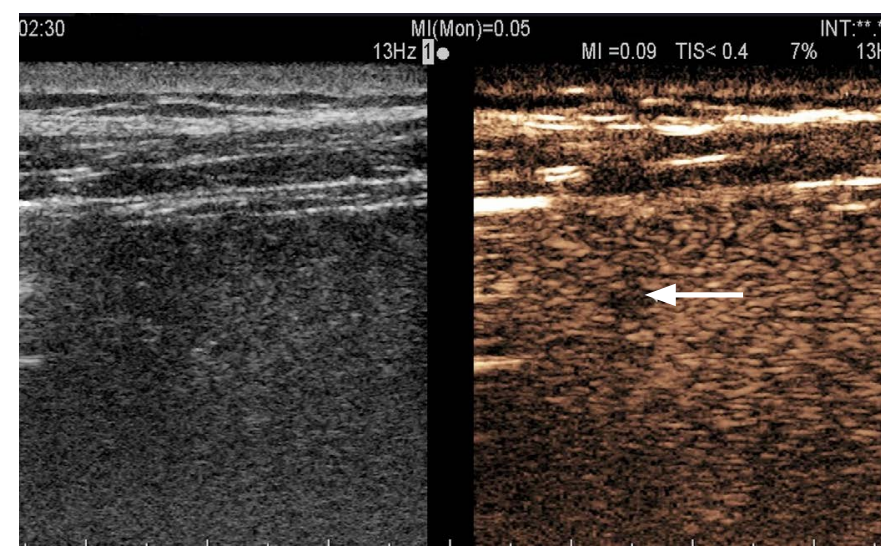

C

Fig. 6. A 68-year-old man with alcoholic liver cirrhosis.

A. A 7-mm subcapsular nodule (arrow) is seen during a surveillance ultrasonography. B. The whole nodule is hyper-enhancing (arrow) in the arterial phase. C. The nodule shows mild and late washout (arrow) in the late phase, 2 minutes 30 seconds after the injection of the contrast. Due to a size smaller than $10 \mathrm{~mm}$, it was classified as LR-4 (a nodule with high probability for hepatocellular carcinoma). Ultrasound-guided biopsy of the nodule confirmed a welldifferentiated hepatocellular carcinoma.

colorectal cancer after chemotherapy, it is recommended to replace unenhanced US with CEUS in the follow-up of patients with liver metastases [4].

CEUS with SonoVue has received approval for pediatric hepatic use in the United States, where it is available under the name of Lumason, but not yet in Europe, where it is still used off-label. The adverse events encountered in pediatric patients and the financial benefits of reducing the number of $\mathrm{CT}$ and MR examinations performed were recently reviewed in a series of 305 pediatric patients [26]. No immediate adverse reactions occurred. Delayed and transient tachycardia, and both were asymptomatic. The potential cost savings of CEUS were $\$ 74$ per examination over CT and \$180 over MR. In a retrospective survey analysis of 948 were recorded [27].

EFSUMB has released a position statement regarding the role of CEUS in pediatric practice, emphasizing that the literature has proven the feasibility, safety, and high diagnostic accuracy of CEUS regarding the characterization of FLLs, similar to CE-CT and CE-MR [28].

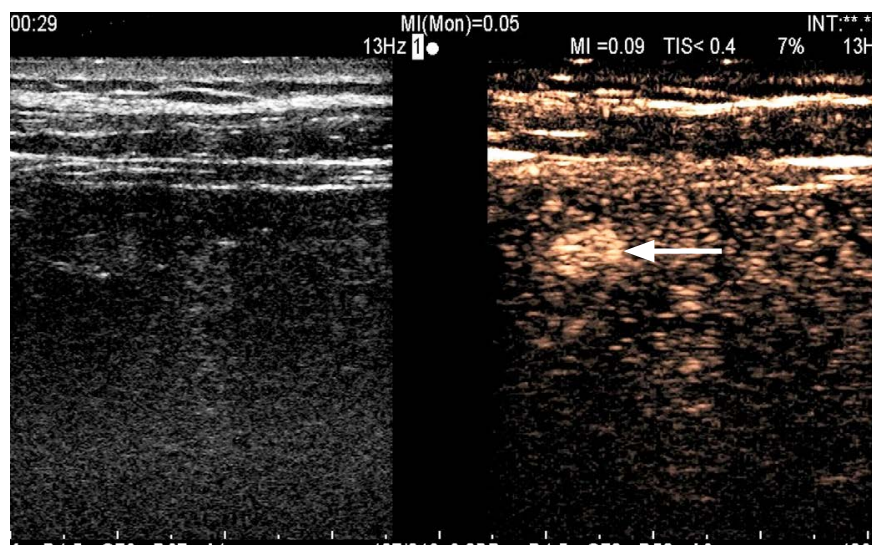

B 


\section{Ablative Treatment and Follow-up of Liver Tumors}

CEUS with SonoVue has been effectively used for guiding the percutaneous treatment of malignant liver tumors that are invisible or poorly visualized in traditional gray-scale US $[29,30]$. In a series of 60 hepatic malignancies, the insertion of the radiofrequency electrodes was guided by CEUS in all lesions, and immediately after

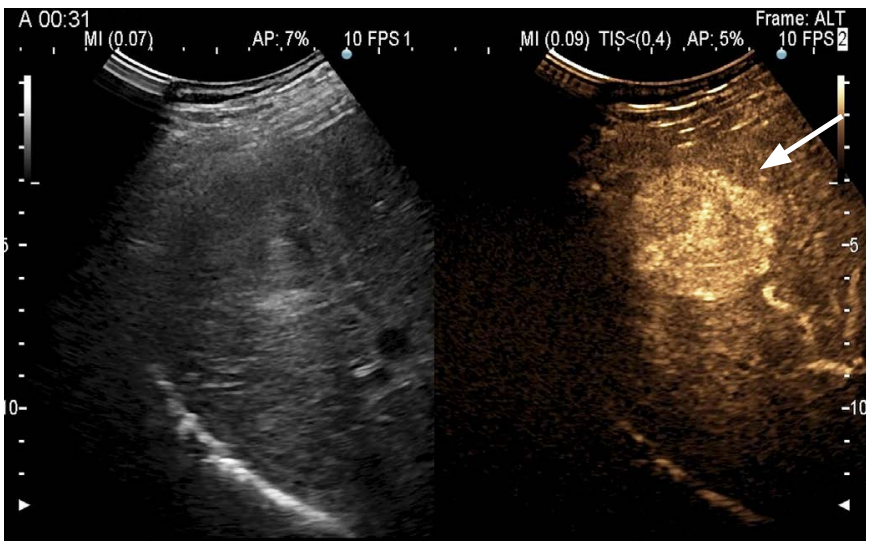

A

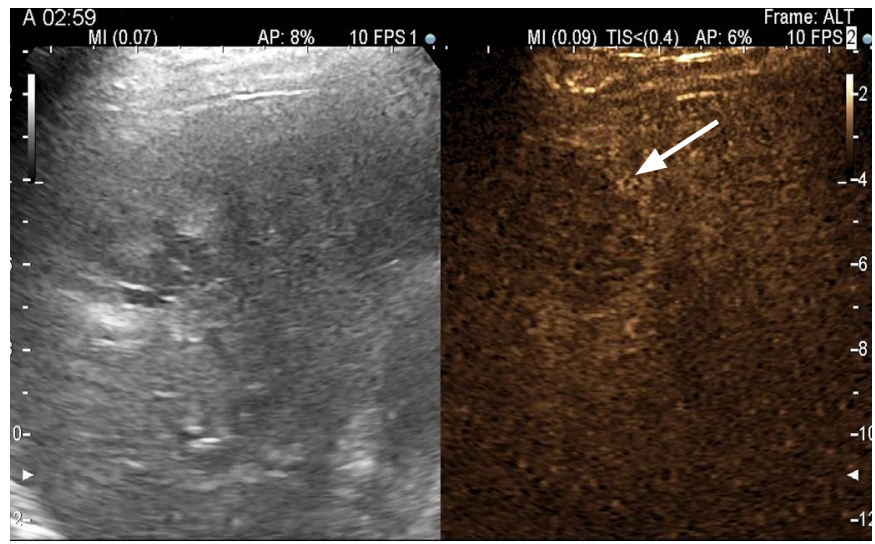

B

Fig. 7. A nodule $3 \mathrm{~cm}$ in size in a 72-year-old woman with liver cirrhosis.

A. The nodule is hyper-enhancing (arrow) in the arterial phase. B. The nodule shows mild and late washout (arrow), which is typical for hepatocellular carcinoma. It was classified as LR-5 (definitely hepatocellular carcinoma).

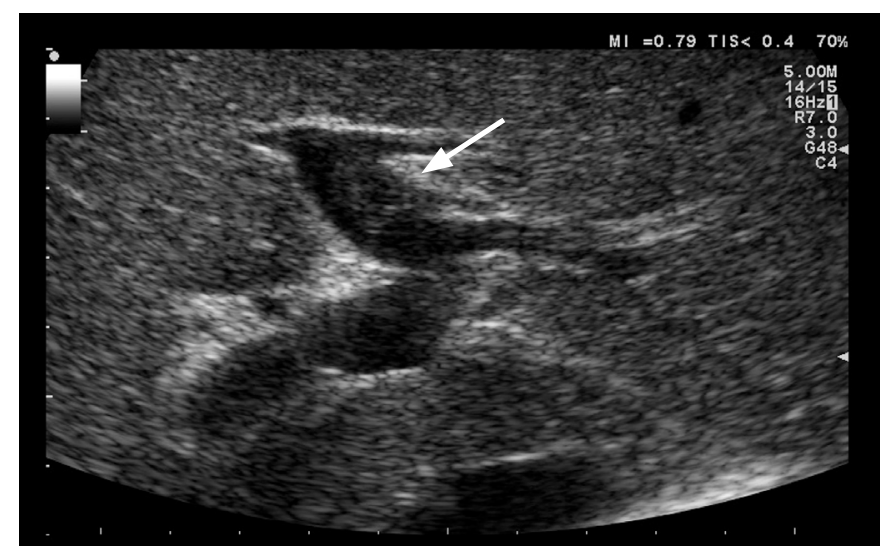

A

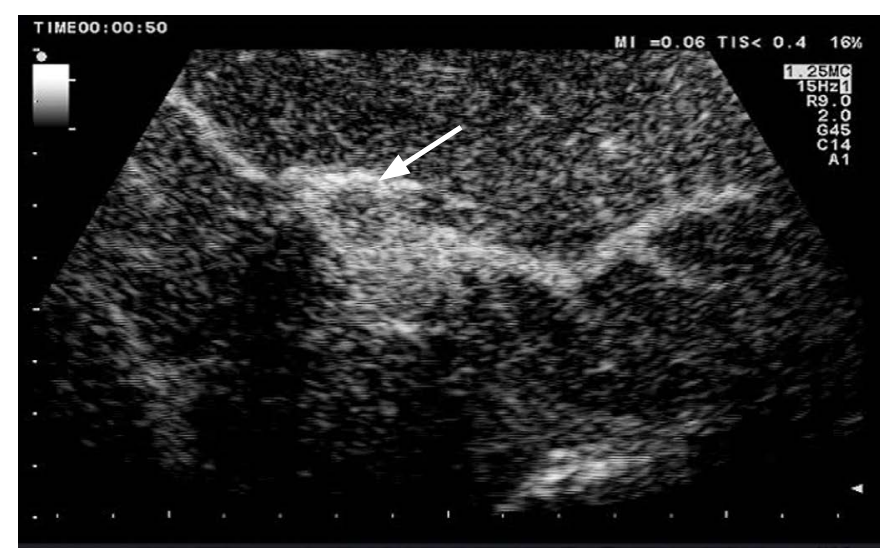

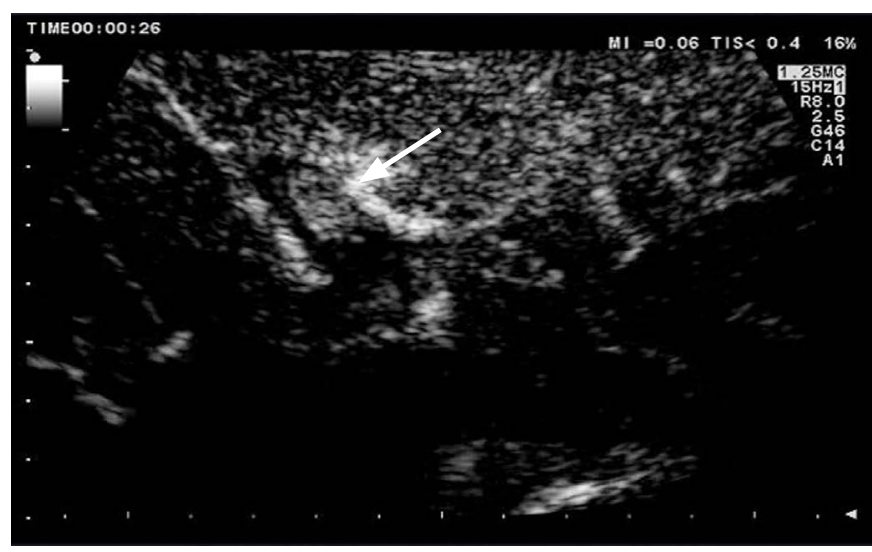

B

Fig. 8. A 75-year-old man with liver cirrhosis and hepatocellular carcinoma.

A. Unenhanced ultrasound shows a thrombus (arrow) in the left branch of the portal vein. B. The thrombus shows enhancement (arrow) in the arterial phase. C. In the late phase, there is washout of the contrast (arrow), allowing the diagnosis of a neoplastic thrombus (i.e., a tumor in the vein) following the contrast-enhanced ultrasonography Liver Imaging Reporting and Data System classification (LR-5V). 


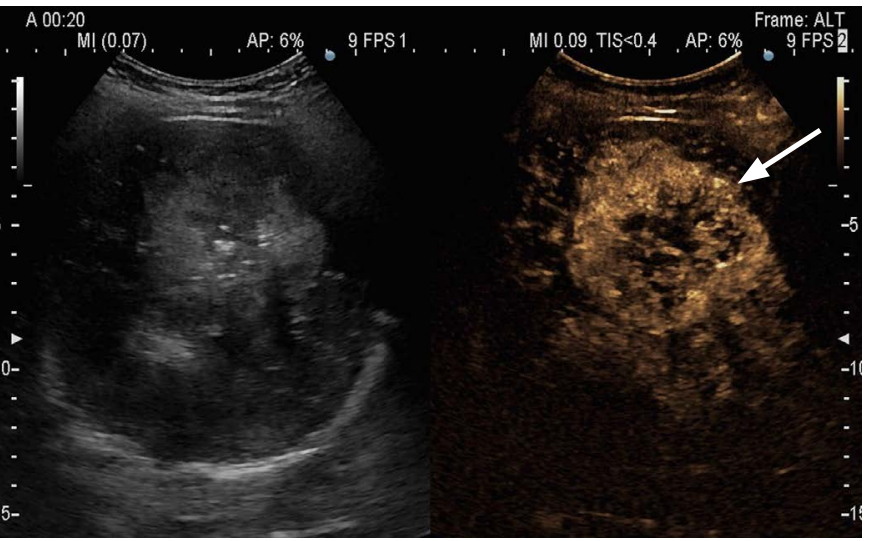

A

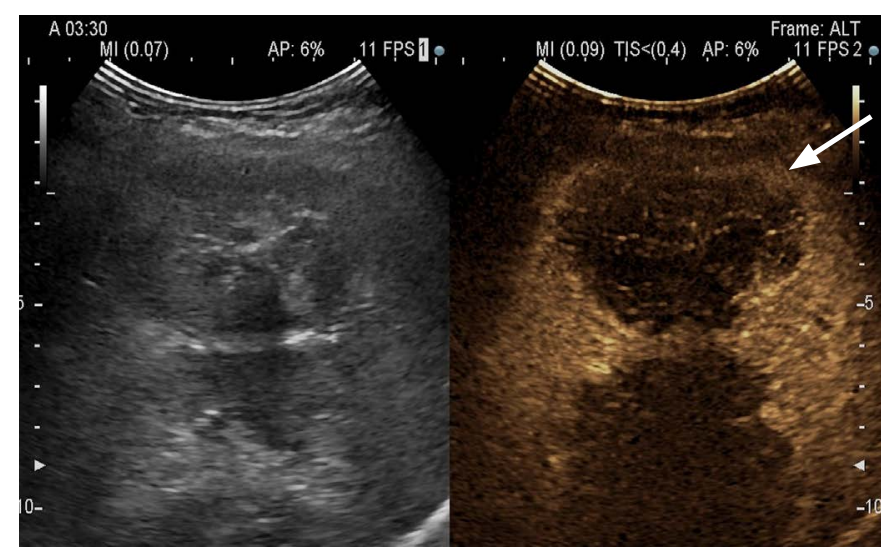

C

the ablation the primary effectiveness rate was $100 \%$, as assessed using CE-MR [30].

A lesion that has been successfully ablated shows no enhancement after contrast administration, whereas residual viable HCC or metastases show the typical pattern of enhancement followed by washout (Fig. 10). However, an enhancing marginal rim can be seen during the first week after ablation, due to an inflammatory reaction that starts soon after the treatment $[29,31]$. This hyper-enhancing halo is usually regular, approximately $1.0 \mathrm{~cm}$ thick, and does not show washout in the late phase [29].

A retrospective study that enrolled 92 patients with 94 tumors showed that the diagnostic accuracy of postprocedural CEUS in the early evaluation of liver tumors following thermal ablation was comparable to both CEUS and CE-CT performed at 24 hours. Thus, it is suggested that postprocedural CEUS may be used to detect and retreat residual viable tissue in the same ablative session [32].

A cost-effectiveness analysis performed in a series of 93 patients with 148 HCCs indicated that the use of intra-procedural CEUS in comparison with standard treatment reduced the costs by $21.9 \%$, because the use of the contrast allowed the detection of incomplete

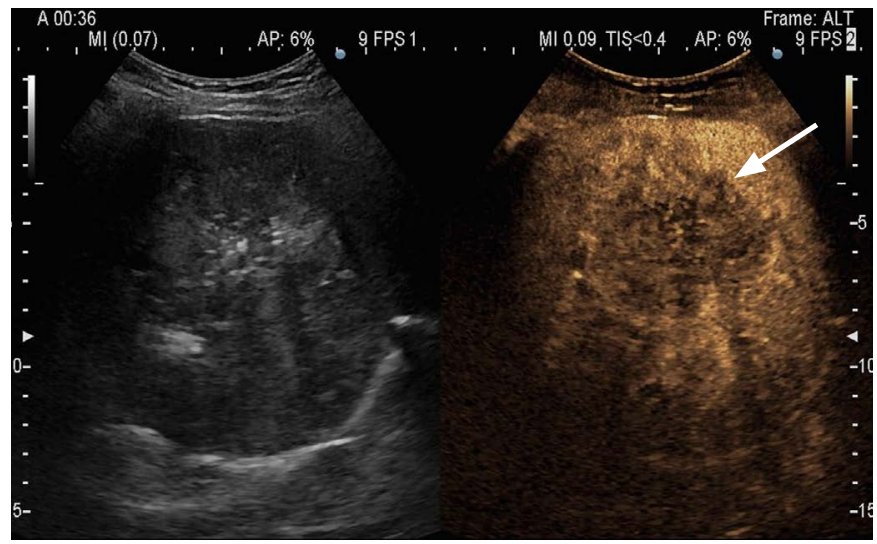

B

Fig. 9. A 45-year-old woman with colon cancer and a synchronous metastasis.

A. It shows early enhancement (arrow) in the arterial phase, 20 seconds after the contrast injection. B. There is a very rapid washout (arrow) in the arterial phase, 36 seconds after the contrast injection. C. In the late phase, the contrast looks completely washed out (arrow).

ablation, meaning that additional treatment could be performed during the same session [33].

In the follow-up of ablated tumors, CE-CT and CE-MR are the gold standard for the assessment of therapeutic efficacy because of their high diagnostic accuracy and the possibility of a thorough evaluation of potential metastatic sites [29]. However, CEUS can be used when CE-CT or CE-MR is inconclusive or contraindicated [29].

In a series of 588 patients with completely ablated tumors who were followed up serially, using alternating CEUS and CE-CT every 3 months for 2 years, recurrences developed in the same segment of the ablated lesion in $72 \%$ of cases; thus, CEUS proved to be effective despite the limited field of view in the arterial phase when compared to CE-CT and CE-MR [34]. Based on these findings, the authors suggested that including CEUS in patients' follow-up may reduce the number of CE-CT and CE-MR examinations, thereby reducing costs and risks.

\section{Diffuse Liver Disease}

CEUS with SonoVue has been used for the assessment of liver 


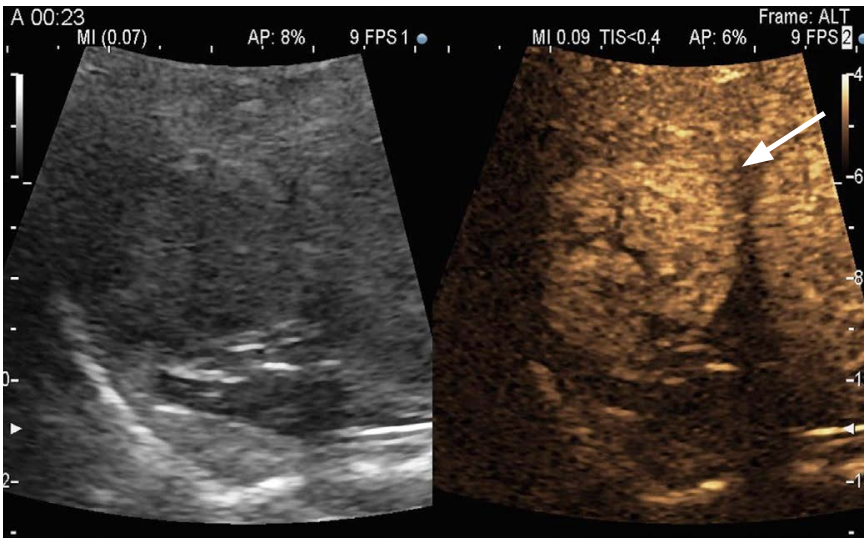

A

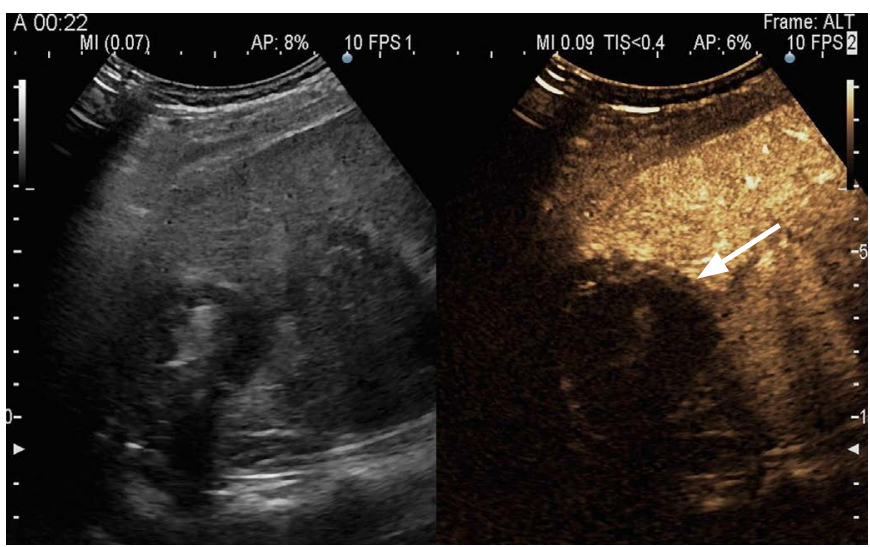

C

disease severity in some studies that have evaluated, using dedicated software, the arrival time of the contrast in the hepatic veins. The rationale is that the capillarization of the sinusoids, together with the presence of arterio-venous and porto-venous shunts, leads to a decrease in the hepatic vein arrival time (HVAT) in patients with cirrhosis. In a study of 160 consecutive patients with cirrhotic $(n=78)$ and noncirrhotic liver disease $(n=82)$ and 14 healthy controls, it was shown that HVAT was lower in cirrhotic patients than in noncirrhotic patients and controls: $15.0 \pm 2.8,21.5 \pm 3.4$, and $25.6 \pm 4.7$ seconds, respectively $(P<0.05)$ [35]. A cutoff value of 17 seconds excluded liver cirrhosis with $91.1 \%$ sensitivity and $93.6 \%$ specificity. In another study performed to identify patients with portal hypertension, the HVAT was significantly shorter among the patients with cirrhosis than in controls $(30.5 \pm 3.3$ vs. $13.3 \pm 3.2$ seconds, $\mathrm{P}<0.001)$ and a HVAT $<14$ seconds identified patients with clinically significant portal hypertension (area under the receiving operator characteristic curve, 0.95; sensitivity, 90.0\%; specificity, 86.7\%) [36].

Despite these interesting results, it should be emphasized that there is a large overlap between the pre-cirrhotic and cirrhotic

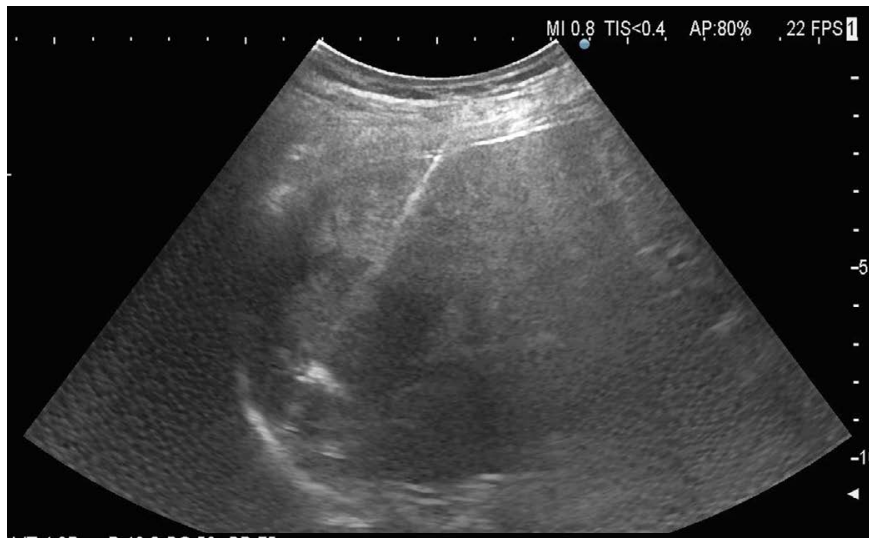

B

Fig. 10. Hepatocellular carcinoma in a 72-year-old man with chronic hepatitis $\mathrm{C}$ and Child $\mathrm{A}$ liver cirrhosis.

A. The figure shows hyper-enhancement (arrow) of the mass in the arterial phase. B. Percutaneous microwave ablation of the hepatocellular carcinoma performed at a power of $100 \mathrm{~W}$ delivered by the antenna for 10 minutes. The figure shows the microwave antenna correctly positioned in the mass. C. Twenty-four hours after treatment, contrast-enhanced ultrasonography shows no enhancement (arrow) of the mass, indicating that the lesion had been effectively ablated.

stages of disease.

\section{Conclusion}

CEUS with SonoVue is a safe and cost-effective imaging procedure for the characterization of FLLs. It provides a real-time dynamic evaluation of FLLs without radiation exposure and can be used even when CE-CT or CE-MR is contraindicated. In several clinical scenarios, the technique is cost-effective compared to CE-CT or CEMR, while having the same accuracy. CEUS allows an immediate characterization of the majority of FLLs incidentally discovered during unenhanced US, thus avoiding more expensive imaging studies that are not free of potential risks.

Limitations in the use of CEUS include the lack of a large field of view and the necessity of ensuring that an appropriate acoustic window is available. Moreover, the results are dependent on the expertise of the operator.

ORCID: Giovanna Ferraioli: http://orcid.org/0000-0002-6344-697X; Maria Franca Meloni: http://orcid.org/0000-0001-7485-7400 


\section{Conflict of Interest}

Giovanna Ferraioli has served as a speaker for Hitachi Ltd., Philips Medical Systems, and Toshiba Medical Systems.

Maria Franca Meloni has served as a speaker for Bracco.

\section{References}

1. Ehrl D, Rothaug K, Herzog P, Hofer B, Rau HG. "Incidentaloma" of the liver: management of a diagnostic and therapeutic dilemma. HPB Surg 2012;2012:891787.

2. de Rave S, Hussain SM. A liver tumour as an incidental finding: differential diagnosis and treatment. Scand I Gastroenterol Suppl 2002;(236):81-86.

3. Piscaglia F, Bolondi L; Italian Society for Ultrasound in Medicine and Biology (SIUMB) Study Group on Ultrasound Contrast Agents. The safety of Sonovue in abdominal applications: retrospective analysis of 23188 investigations. Ultrasound Med Biol 2006;32:1369-1375.

4. Claudon M, Dietrich CF, Choi BI, Cosgrove DO, Kudo M, Nolsoe CP, et al. Guidelines and good clinical practice recommendations for contrast enhanced ultrasound (CEUS) in the liver: update 2012: a WFUMB-EFSUMB initiative in cooperation with representatives of AFSUMB, AIUM, ASUM, FLAUS and ICUS. Ultraschall Med 2013;34:11-29.

5. Greis C. Quantitative evaluation of microvascular blood flow by contrast-enhanced ultrasound (CEUS). Clin Hemorheol Microcirc 2011;49:137-149.

6. Trillaud H, Bruel JM, Valette PJ, Vilgrain V, Schmutz G, Oyen R, et al. Characterization of focal liver lesions with SonoVue-enhanced sonography: international multicenter-study in comparison to CT and MRI. World J Gastroenterol 2009;15:3748-3756.

7. Strobel D, Bernatik T, Blank W, Schuler A, Greis C, Dietrich CF, et al. Diagnostic accuracy of CEUS in the differential diagnosis of small $(\leq 20 \mathrm{~mm})$ and subcentimetric $(\leq 10 \mathrm{~mm})$ focal liver lesions in comparison with histology: results of the DEGUM multicenter trial. Ultraschall Med 2011;32:593-597.

8. Sporea I, Badea R, Popescu A, Sparchez Z, Sirli RL, Danila M, et al. Contrast-enhanced ultrasound (CEUS) for the evaluation of focal liver lesions: a prospective multicenter study of its usefulness in clinical practice. Ultraschall Med 2014;35:259-266.

9. Tranquart F, Correas JM, Ladam Marcus V, Manzoni P, Vilgrain $V$, Aube $C$, et al. Real-time contrast-enhanced ultrasound in the evaluation of focal liver lesions: diagnostic efficacy and economical issues from a French multicentric study. J Radiol 2009;90(1 Pt 2):109-122.

10. Friedrich-Rust M, Klopffleisch T, Nierhoff J, Herrmann E, Vermehren J, Schneider MD, et al. Contrast-enhanced ultrasound for the differentiation of benign and malignant focal liver lesions: a metaanalysis. Liver Int 2013;33:739-755.

11. Westwood M, Joore M, Grutters J, Redekop K, Armstrong N, Lee
$K$, et al. Contrast-enhanced ultrasound using SonoVue(R) (sulphur hexafluoride microbubbles) compared with contrast-enhanced computed tomography and contrast-enhanced magnetic resonance imaging for the characterisation of focal liver lesions and detection of liver metastases: a systematic review and cost-effectiveness analysis. Health Technol Assess 2013;17:1-243.

12. Romanini L, Passamonti M, Aiani L, Cabassa P, Raieli G, Montermini I, et al. Economic assessment of contrast-enhanced ultrasonography for evaluation of focal liver lesions: a multicentre Italian experience. Eur Radiol 2007;17 Suppl 6:F99-F106.

13. Guang $Y$, Xie L, Ding H, Cai A, Huang Y. Diagnosis value of focal liver lesions with SonoVue(R)-enhanced ultrasound compared with contrast-enhanced computed tomography and contrast-enhanced MRI: a meta-analysis. J Cancer Res Clin Oncol 2011;137:15951605.

14. Bartolotta TV, Taibbi A, Picone D, Anastasi A, Midiri M, Lagalla R. Detection of liver metastases in cancer patients with geographic fatty infiltration of the liver: the added value of contrast-enhanced sonography. Ultrasonography 2017;36:160-169.

15. Jang JK, Jang HJ, Kim JS, Kim TK. Focal fat deposition in the liver: diagnostic challenges on imaging. Abdom Radiol (NY) 2017;42:1667-1678.

16. Ferraioli G, Dellafiore C, Meloni MF, Calliada F, Filice C. A review of the appropriateness of the current Italian guidelines for noninvasive imaging assessment of focal liver lesions. J Gastrointestin Liver Dis 2015;24:491-497.

17. Bruix J, Sherman M; American Association for the Study of Liver Diseases. Management of hepatocellular carcinoma: an update. Hepatology 2011;53:1020-1022.

18. European Association for the Study of the Liver; European Organisation for Research and Treatment of Cancer. EASLEORTC clinical practice guidelines: management of hepatocellular carcinoma. J Hepatol 2012;56:908-943.

19. Vilana R, Forner A, Bianchi L, Garcia-Criado A, Rimola J, de Lope $C R$, et al. Intrahepatic peripheral cholangiocarcinoma in cirrhosis patients may display a vascular pattern similar to hepatocellular carcinoma on contrast-enhanced ultrasound. Hepatology 2010;51:2020-2029.

20. Italian Association for the Study of the Liver (AISF); AISF Expert Panel; AISF Coordinating Committee, Bolondi L, Cillo U, Colombo M, et al. Position paper of the Italian Association for the Study of the Liver (AISF): the multidisciplinary clinical approach to hepatocellular carcinoma. Dig Liver Dis 2013;45:712-723.

21. Filice C, Calliada F, De Masi S, Sampaolo L, Morciano C, Mele A, et al. Italian guidelines for noninvasive imaging assessment of focal liver lesions: development and conclusions. Eur J Gastroenterol Hepatol 2011;23:343-353.

22. NICE. SonoVue (sulphur hexafluoride microbubbles): contrast agent for contrast-enhanced ultrasound imaging of the liver [Internet]. 
London: National Institute for Health and Care Excellence; 2017 [cited 2017 Apr 9]. Available from: https://www.nice.org.uk/ guidance/dg5/chapter/1-Recommendations.

23. de Sio I, Iadevaia MD, Vitale LM, Niosi M, Del Prete A, de Sio $C$, et al. Optimized contrast-enhanced ultrasonography for characterization of focal liver lesions in cirrhosis: a single-center retrospective study. United European Gastroenterol J 2014;2:279287.

24. Aube C, Oberti F, Lonjon J, Pageaux G, Seror O, N'Kontchou G, et al. EASL and AASLD recommendations for the diagnosis of HCC to the test of daily practice. Liver Int 2017 Mar 27 [Epub]. https://doi.org/10.1111/liv.13429.

25. Piscaglia F, Wilson SR, Lyshchik A, Cosgrove D, Dietrich CF, Jang HJ, et al. American College of Radiology Contrast Enhanced Ultrasound Liver Imaging Reporting and Data System (CEUS LI-RADS) for the diagnosis of hepatocellular carcinoma: a pictorial essay. Ultraschall Med 2017;38:320-324.

26. Yusuf GT, Sellars ME, Deganello A, Cosgrove DO, Sidhu PS. Retrospective analysis of the safety and cost implications of pediatric contrast-enhanced ultrasound at a single center. AJR Am J Roentgenol 2017;208:446-452.

27. Riccabona M. Application of a second-generation US contrast agent in infants and children: a European questionnaire-based survey. Pediatr Radiol 2012;42:1471-1480.

28. Sidhu PS, Cantisani V, Deganello A, Dietrich CF, Duran C, Franke D, et al. Role of contrast-enhanced ultrasound (CEUS) in paediatric practice: an EFSUMB position statement. Ultraschall Med 2017:38:33-43.

29. Meloni MF, Smolock A, Cantisani V, Bezzi M, D'Ambrosio F, Proiti $M$, et al. Contrast enhanced ultrasound in the evaluation and percutaneous treatment of hepatic and renal tumors. Eur J Radiol
2015;84:1666-1674.

30. Dong Y, Wang WP, Gan YH, Huang BJ, Ding H. Radiofrequency ablation guided by contrast-enhanced ultrasound for hepatic malignancies: preliminary results. Clin Radiol 2014;69:1129-1135.

31. Guibal A, Bertin C, Egels S, Savier E, Grenier PA, Lucidarme 0 . Contrast-enhanced ultrasound (CEUS) follow-up after radiofrequency ablation or cryoablation of focal liver lesions: treated-area patterns and their changes over time. Eur Radiol 2013;23:1392-1400.

32. Meloni MF, Andreano A, Franza E, Passamonti M, Lazzaroni S. Contrast enhanced ultrasound: should it play a role in immediate evaluation of liver tumors following thermal ablation? Eur J Radiol 2012:81:e897-e902.

33. Mauri G, Porazzi E, Cova L, Restelli U, Tondolo T, Bonfanti M, et al. Intraprocedural contrast-enhanced ultrasound (CEUS) in liver percutaneous radiofrequency ablation: clinical impact and health technology assessment. Insights Imaging 2014;5:209-216.

34. Catalano O, Izzo F, Vallone P, Sandomenico F, Albino V, Nunziata A, et al. Integrating contrast-enhanced sonography in the follow-up algorithm of hepatocellular carcinoma treated with radiofrequency ablation: single cancer center experience. Acta Radiol 2015;56:133142.

35. Abbattista T, Ridolfi F, Consalvo GT, Brunelli E. Hepatic vein arrival time for diagnosis of liver cirrhosis: a 10-year single-center experience with contrast-enhanced sonography. J Ultrasound Med 2016;35:2121-2128.

36. Kim MY, Suk KT, Baik SK, Kim HA, Kim YJ, Cha SH, et al. Hepatic vein arrival time as assessed by contrast-enhanced ultrasonography is useful for the assessment of portal hypertension in compensated cirrhosis. Hepatology 2012;56:1053-1062. 